\title{
FIRST-PRINCIPLES STUDY OF MOLECULAR ADSORPTION OF HYDROGEN/S ON Co- ADATOM GRAPHENE
}

\author{
Nurapati Pantha, Saroj Thapa, Narayan Prasad Adhikari* \\ Central Department of Physics, Tribhuvan University, Kathmandu, Nepal \\ ${ }^{*}$ Corresponding author: npadhikari@gmail.com
}

(Received: February 29, 2020; Revised: May 16, 2020; Accepted: May 29, 2020)

\begin{abstract}
The study of graphene and its allotropes help to understand fundamental science and their role in the industry. The adsorption of transition metal adatom on mono-layer graphene can tune the geometrical, electronic, and magnetic properties of the material according to the requirement for the practical applications. In the present work, the geometrical stability, electronic and magnetic properties, and also the redistribution of electronic charge of single cobalt atom (Co) adsorbed graphene with reference to pure graphene have been investigated to develop a model system for the effective storage of hydrogen. The density functional theory (DFT) based first-principles calculations by incorporating van der Waals (VDW) interactions within DFT-D2 levels of approximation implemented in the quantum ESPRESSO package was used. The band structure and density of states of cobalt-adatom graphene show that the material is metallic and magnetic with a total magnetic moment of $1.55 \mu_{\mathrm{B}}$. The change in the electronic distribution of Co-adatom graphene has been found favorable for adsorbing molecular hydrogen/s with greater strength. The increasing number of adsorbed molecular hydrogen/s ( $\mathrm{n}=1$ to 7$)$ onto the substrate shows varying binding energy per hydrogen molecule, high enough at low concentration $(n=1,2$, and 3$)$, and then decreases slowly on increasing the value of $n$. The nature of adsorption and binding energy per hydrogen molecule (with a range of $0.116-0.731 \mathrm{eV} / \mathrm{H}_{2}$ ) are found useful to meet a standard target for hydrogen storage in such materials.
\end{abstract}

Keywords: Adatom-graphene, Charge transfer, Energy storage, Density-functional theory, van der Waal's interaction

\section{INTRODUCTION}

Graphene, an allotrope of carbon, is characterized by one atom thick sheet of $\mathrm{sp}^{2}$ bonded carbon atoms that is tightly packed into a two-dimensional (2D) honeycomb lattice (Neto et al., 2006). Since, the carbon materials of different dimensions like fullerenes, carbon nanotubes, nanoribbons, graphite, etc. can be considered to be built up from graphene, it is said to be the mother of graphitic materials. After the successful isolation of monolayer graphene in 2004 (Novoselov et al., 2005), graphene and related compounds have become one of the frontier topics to investigate its intriguing properties (Geim \& Novoselov, 2007). Graphene has many interesting properties like highly transparent, stretchable, strong, and very high conductivity of heat and electricity (Geim, 2009). Furthermore, graphene is described as zero bandgap semiconductors (Neto et al., 2009), because the conduction band and valence band meet at the Fermi level. The tunable bandgap of graphene is one of the interesting properties for its semiconductor applications (Han et al., 2007).

The deliberate addition of impurities on the pure system, called functionalization, aims for the advancement of technology and applications. The functionalization of graphene and other nanosystems with metal-atoms, molecules, and functional groups is a promising approach to controllably modify their properties like the electronic structures, bandgap, the density of states, and the geometrical structures (Paudel et al., 2018). The adsorption of a metal adatom on graphene and carbon nanostructures has become one of the widely searched topics. Chan and coworkers studied 12 different metal adatoms on graphene by using first-principles density functional theory-based calculations in VASP (Chan et al., 2008). The authors included the adatoms from the alkali metals, transition metals, and noble metals, and discuss their geometries, band structures, charge transfer, and other electronic/magnetic properties. Using DFT calculations, the transition metal adatoms from the $3 \mathrm{~d}$ series on graphene and single-walled carbon nanotubes (SWCNTs) have been studied by Valencia et al. (2010). The study revealed both the weak and strong type of interactions between the adatoms and the graphitic surfaces. Electronic configurations of metal atoms helped to describe such bindings; the atoms having valence shell totally or half-filled, were found weakly bound and the atoms with partially filled valence shell were strongly bound.

Hydrogen is considered as a clean source of energy as it emits only water vapor while burning with oxygen. It is dominantly available in the earth and also in the extraterrestrial planets either in the gaseous form or as a component of other compounds. However, one of the major problems to use hydrogen as gaseous fuel is its effective and safe storage. The traditional techniques to store hydrogen and other fuel gases in the compressed form at very high pressure and in the liquefied form at 
very low temperatures are not user friendly due to space, weight, economy, and safety issues (Chen et al., 1999). Adsorption of molecular hydrogen with suitable strength and energy density could be one of the prominent alternatives, and this process in the porous materials like carbon and other nano-materials was highly recommended (Pan et al., 2004; Zhang et al., 2019). Pure graphene and its derivative are less reactive towards the inert gases like hydrogens and do not provide enough strength to hold them. It is common practice to use some techniques like functionalization, use of stress, changing size, orientation and geometry of the material to enhance the content of hydrogen and binding strength to meet the standard target (Durgun et al., 2008; Zhou \& Williams, 2011; Jain \& Kandasubramanian, 2020).

In the present work, the geometries, electronic structure, and magnetic properties of single Co adatom graphene comparing to pure graphene were studied. The Co adatom-graphene was used as a substrate to adsorb molecular hydrogen/s in different concentrations, starting from $n=1$ to $n=7$, which intends to develop a model for the effective storage of hydrogen as a gaseous fuel at operating conditions. Here the effective storage incorporates the proper binding strength to hydrogen and higher value of energy (hydrogen) density. Binding strength should be high enough to hold the molecular hydrogen/s at normal (storage) condition, however, it should be released at operating condition (pressure/temperature) (Bhatia \& Myers, 2006). A high value of energy density implies the higher content of hydrogen (fuel) in the specific volume.

\section{MATERIALS AND METHODS}

The spin-polarized density-functional theory (DFT) calculations (Hohenberg \& Kohn, 1964; Kohn \& Sham, 1965) were performed to study the effect of single Coadatom on mono-layer graphene intending to develop effective hydrogen storage medium. The plane wave selfconsistent field (PWSCF) implementation of DFT-D2 calculations in quantum espresso package (Giannozzi et al., 2009) was used to incorporate van der Walls interaction over the standard DFT calculations. The interaction between the ion-cores and the valence electrons is accounted for by the ultra-soft pseudopotentials. In the pseudo-potential description of the electronic configurations $1 \mathrm{~s}^{2}, \quad 2 \mathrm{~s}^{2} 2 \mathrm{p}^{2}$, and $4 \mathrm{~s}^{2} 3 \mathrm{~d}^{7}$ of hydrogen, carbon, and cobalt atoms, respectively are treated explicitly as valence (electrons). The exchange and correlations parts of electron-electron interactions are treated by generalized gradient approximation (GGA) as suggested by Perdew-Burke-Ernzerhof (PBE) (Perdew et al., 1996).

The structure was treated in the graphene supercell using periodic boundary conditions. The stable size of graphene for the adsorption of a single Co atom was checked by calculating the binding energy of Co in $2 \times 2,3 \times 3$, and $4 \times 4$ supercells of graphene. To obtain the optimized geometries, the systems were fully relaxed using BFGS (Broyden-Fletcher-Goldfarb-Shanno) (Head \& Zerner, 1985) scheme until the total energy change becomes less than $10^{-4}$ Ry between two consecutive scf steps and force acting goes below the $10^{-3} \mathrm{Ry} / \mathrm{Bohr}$. For the selfconsistent total energy calculations, the Brillouin zone of graphene was sampled by using the Monkhorst-Pack scheme (Monkhorst \& Pack, 1976) with the appropriate number of k-points, estimated from the convergence test. The 'Marzarri-vanderbilt' (Marzari et al., 1999) smearing of width $0.001 \mathrm{Ry}$ is used to assist the convergence. Furthermore, 'david' diagonalization with mixing factor 0.6 has been used for self-consistency.

Convergence test is a usual method to select the simulating parameters in the DFT level of calculations (Pantha \& Adhikari, 2014). A similar method of calculations has been employed to choose plane wave cutoff values of electronic wave-functions and charge density of $35 \mathrm{Ry}$ and $350 \mathrm{Ry}$, respectively. The convergence test is also useful to select the lattice constant and a number of the k-points and the same method has been used to select the lattice constant $(\mathrm{a}=4.65$ Bohrs $)$, and the number of $\mathrm{k}$ point mesh $\left(\mathrm{n}_{\mathrm{kx}}=20, \mathrm{n}_{\mathrm{ky}}=20, \mathrm{n}_{\mathrm{kz}}=1\right)$ for the unit cell structure of graphene. The value of lattice constant calculated by this method agrees well with the experiment (Neto et al., 2009). Using the information of binding energy, we predict the most stable site for the adsorption of single Co atom in $4 \times 4$ supercell of graphene, and the same geometry was used to adsorb the varying number of hydrogen molecules. The information from the charge difference/transfer calculations was to analyze the nature of the interaction between the substrate (modified graphene) and the gaseous molecules.

\section{RESULTS AND DISCUSSION}

The minimum energy structures of Co-adsorbed graphene and pure graphene have been observed via relaxation. Also, the total energy for isolated Co atom has been found through self-consistent calculations. The binding energy of Co on different sizes and sites of graphene supercells has then been calculated, by using equation (1), to analyze the size and site dependency. The higher the binding energy implies the more strongly the atom is held on the substrate.

$$
\mathrm{E}=\mathrm{E}_{\mathrm{a}}+\mathrm{E}_{\mathrm{g}}-\mathrm{E}_{\mathrm{ag}}
$$

Here, $E_{a}$ is the total energy of an isolated adatom (Co in our case), $E_{g}$ is the total energy of isolated graphene, and $E_{\text {ag }}$ is the total energy of the adatom graphene system (total energy per adatom).

The adsorption of Co has been tested at three high symmetry sites; (i) top (T): directly above the carbon 
atom, (ii) hollow $(\mathrm{H})$ : at the center of the hexagon, and (iii) bridge (B): at the midpoint of a $\mathrm{C}-\mathrm{C}$ bond. The relaxed structures of these configurations for $4 \times 4$ supercells are shown in Fig. 1. Table 1 shows the binding energies of $\mathrm{Co}$ at $4 \times 4$ supercell of graphene, which implies that the hollow site is the most favorable site for its adsorption. While going through the size of the substrate, the binding energy values of Co have been found as $1.37 \mathrm{eV}, 1.22 \mathrm{eV}$, and $1.26 \mathrm{eV}$ in $2 \times 2,3 \times 3$, and $4 \times 4$ supercells (at the hollow site), respectively. This shows that the binding energy varies very slightly with reference to the supercell size after $3 \times 3$. It is then logical to use $4 \times 4$ supercells as the host substance to hold adatom $\mathrm{Co}$ and upcoming hydrogen/s.

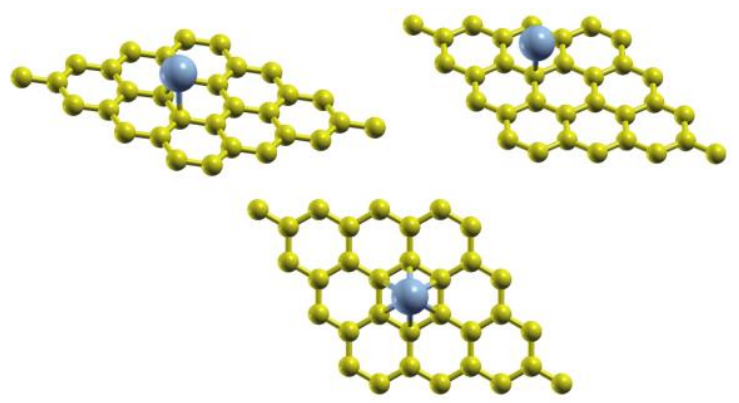

Fig. 1. Slant-view of optimized geometry of single cobalt atom adsorbed on three different high symmetric sites Bridge (B), Top (T), and Hollow (H) of graphene (The structures have been generated by using a software (Xcrysden) based on parameters taken from the convergence test)

Table 1 lists the binding energy of Co-adatom on graphene $(\mathrm{E})$, its vertical height from the graphene layer (h), the actual distance of adatom and nearest carbon/s $\left(\mathrm{d}_{\mathrm{AC}}\right)$, and maximum distortion in graphene plane due to adsorption of adatom with reference to the initial position of graphene $(\Delta d)$, respectively, for the adsorption of cobalt at three different sites. The binding energy of cobalt at the hollow site $(1.26 \mathrm{eV})$ was found the highest among the tested sites. It means the hollow site is the most favorable place of $4 \times 4$ supercell of graphene (containing 32 carbon atoms) which comes in good agreement with the previous reports (Sevincli et al., 2008; Yagi et al., 2004) where Sevincli and co-workers mention the binding energy as $(1.27 \mathrm{eV})$. The adsorption energies at $\mathrm{B}$ and $\mathrm{T}$ sites from the present calculations, on the other hand, were significantly smaller by $0.74 \mathrm{eV}$ (for B site) and $0.73 \mathrm{eV}$ (for $\mathrm{T}$ site) than that for the $\mathrm{H}$-site. The binding energy values also imply that the barrier height to transit $\mathrm{Co}$ atom from $\mathrm{H}$ site to the other two symmetry sites was high (in the order of $0.5 \mathrm{eV}$ ). The distance of the cobalt from its nearest carbon atom for $\mathrm{H}, \mathrm{B}$, and $\mathrm{T}$ sites was $2.08 \AA 1.96 \AA$ and $2.02 \AA$ respectively. Besides, the vertical heights of the Co from the average graphene layer were $1.47 \AA$, $1.89 \AA$, and $2.07 \AA$ (sites in the same order). This geometry (the smallest vertical height for $\mathrm{H}$ site) signifies that the interaction between the cobalt and carbon atoms is stronger at $\mathrm{H}$ site than that of $\mathrm{B}$ and $\mathrm{T}$ sites. The maximum distortion produced in the plane of the graphene sheet (in the $\mathrm{z}$-direction) due to the adsorption of cobalt at $\mathrm{H}, \mathrm{B}$, and $\mathrm{T}$ sites were found to be $0.040 \AA, 0.074 \AA$, and $0.054 \AA$, respectively.

Table 1. Structural properties for the hollow $(\mathrm{H})$, bridge (B), and top (T) sites of the cobalt adatom on $4 \times 4$ supercell of graphene

\begin{tabular}{|c|c|c|c|c|}
\hline Site & $\mathrm{E}(\mathrm{eV})$ & $\mathrm{H}(\AA)$ & $\mathrm{d}_{\mathrm{AC}}(\AA)$ & $\Delta \mathrm{d}\left(10^{-1} \AA\right)$ \\
\hline Hollow & 1.264 & 1.47 & 2.08 & 0.40 \\
\hline Bridge & 0.740 & 1.89 & 1.96 & 0.74 \\
\hline Top & 0.732 & 2.07 & 2.02 & 0.54 \\
\hline
\end{tabular}

After getting minimum energy structures of pure and adatom graphene, we performed their band structure calculations. Figure 2 (a) shows the calculated band structure of pure graphene. The figure displays that the conduction band and the valence band meet at the Fermi level, and form the conical band structures. The conical structures thus formed are called the Dirac cones and the point where the Dirac cones meet is called Dirac point. Since the conduction band and the valance band meet at the Fermi level (with zero gap), graphene is called zero bandgap semiconductor (Neto et al., 2009).

The band structure of cobalt adsorbed graphene was calculated and results are shown in Fig. 2 (b). The figure shows that the Fermi level shifts above the conical point (towards the conduction band) due to the adsorption of cobalt atom on graphene. Before adsorption, all the states of $\pi$ band were filled and $\pi^{*}$ bands were empty. When the cobalt atom was adsorbed on graphene, some amount of electronic charge transferred from cobalt atom to graphene. Since the $\pi$ band of the graphene was already occupied, it is forced to move towards the $\pi^{*}$ band. The overlapping of the valance band and conduction band implies that the material is metallic in nature (Dahal et al. 2014). Since Figs. 2(b) and 3(b) show conduction and valence bands overlapping to each other, cobalt adatom graphene becomes a metallic system. Also, some extra contribution to the band structure of Co-added graphene was observed over the pure graphene which is consistent with the previous study (Yagi et al., 2004).

The density of states (DOS) describes the available energy orbitals to be occupied by the electrons. The present work shows DOS of pure graphene for the spin up and spin down perfectly symmetric as depicted in Fig. 3. The figure also displays that the DOS is almost zero at the Fermi level. These results are supported by the well- 
established facts that graphene is non-magnetic in nature and zero-gap semiconductor (Neto et al., 2009).
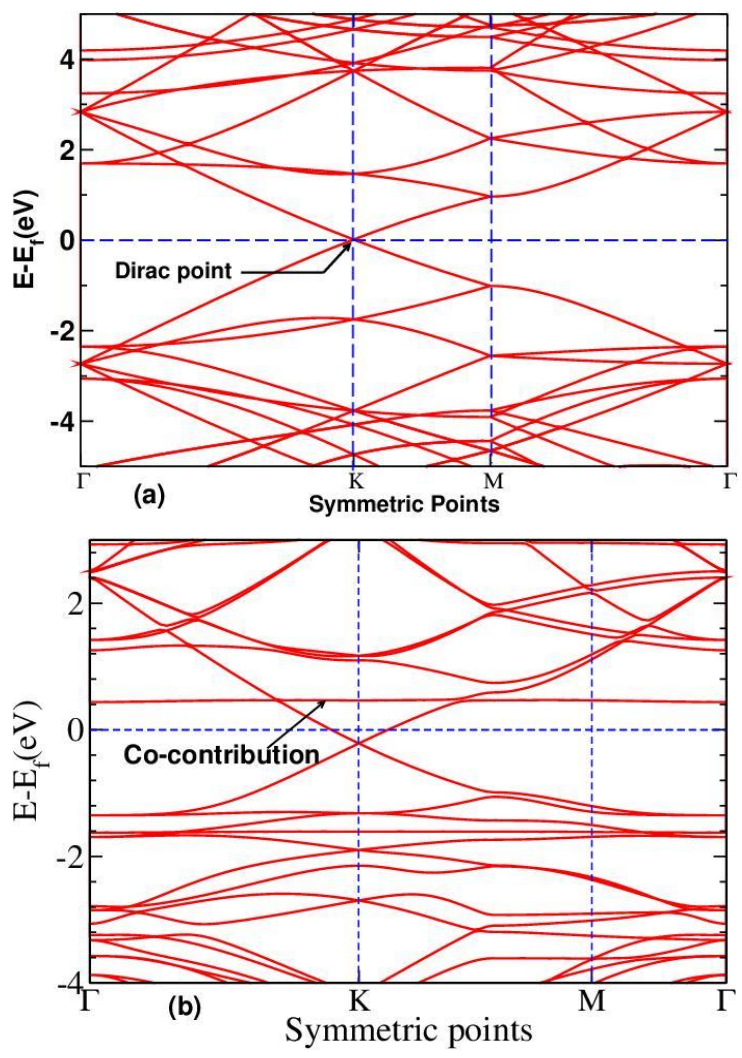

Fig. 2. Band structure of the $4 \times 4$ supercells of pure graphene and cobalt adsorbed graphene along $\Gamma$ - $K$ $M-\Gamma$ path of the Brillouin zone

In the case of cobalt adsorbed graphene, DOS for the spin up and spin down was different. During the interaction of Co atom with graphene, the $4 \mathrm{~d}$ orbitals of cobalt strongly hybridized with the graphene states. Since the Co orbitals lie nearby and below the Fermi-level, the process of hybridization changes the nature of DOS, which ultimately modifies the properties of the material regarding the pure graphene.

Similarly, 4s orbital lies above the Fermi level and was unoccupied (Fig. 4). It claims the possibility of rearrangement of electronic occupation when Co comes closer to the graphene. The electrons with spin down, having larger contributions nearby the Fermi level are the conducting electrons and play an important role in conductivity. The density of states, in this case, is thus different from zero and is the signature of metallic property. Furthermore, the spin polarization of DOS indicated the magnetic nature of the material. Present calculations found the magnitude of total magnetization (of Co-adatom graphene) as $1.55 \mu_{\mathrm{B}}$. The value was different from both of its constituents, graphene $0 \mu_{\mathrm{B}}$ (Paudel et al., 2015) and isolated cobalt $3 \mu_{\mathrm{B}}$ (Kittel, 2015).
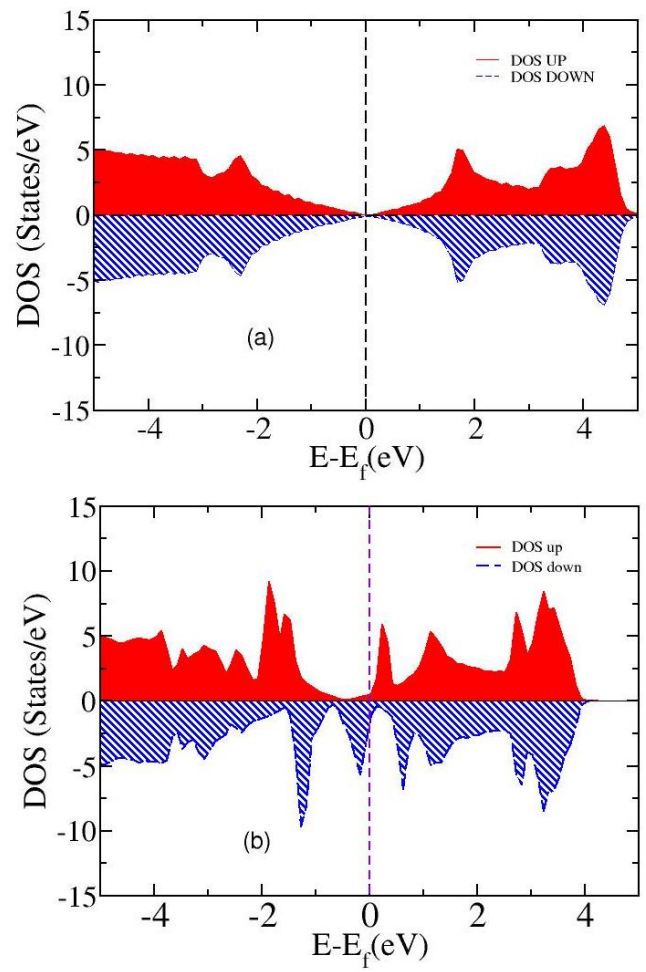

Fig. 3. The density of states for pure graphene (a) and cobalt adsorbed graphene (b).
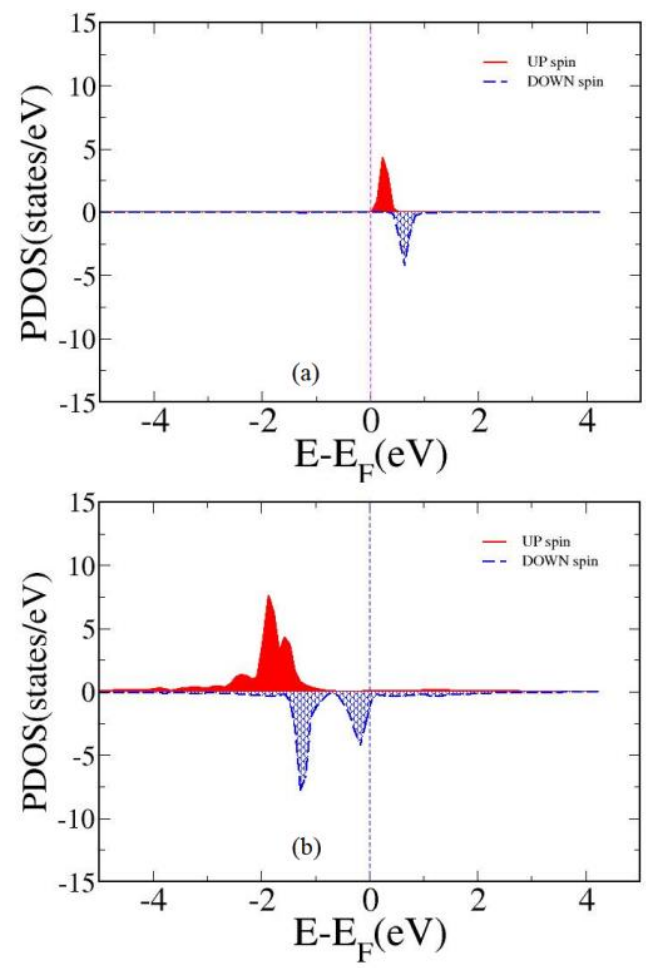

Fig. 4. The density of states (PDOS) for the spin up and spin down of s-orbitals (a) and d-orbitals (b) of cobalt adsorbed on graphene 
In the process of adsorption of an adatom on graphene, there was a rearrangement of electronic charge within the system and also in between the interacting systems. This disturbs the symmetry of charge distribution, polarizes the specimen, and finally transfers a certain amount of charge from one side to another. The study of nature and quantity of charge transfer is important to understand how the adatom and the graphene communicate with each other.

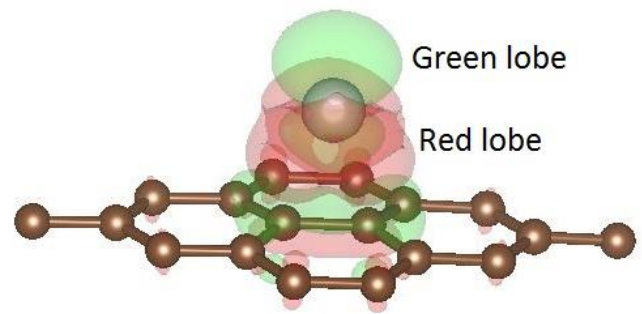

Fig. 5. The charge density difference in between Co added graphene and its constituents (graphene and Co atom), calculated in the same sized cell $(4 \times 4$ supercells of graphene). The green lobes represent the charge depletion and the red lobes represent charge accumulation regions in the iso-surface structures (color online)

The charge density difference between Co adatom graphene and its constituents is shown in Fig. (5). The green lobes (mainly lying far end of cobalt atom from graphene surface) represent the charge depletion regions and red lobes (lying near end of cobalt atom) in the isosurface structures. The depletion of electronic charge at cobalt at the far side of the atom implies that a fraction of electronic charge transfers from the Co atom towards the graphene (substrate). The rearrangement/transfer of charge helps to polarize a gaseous molecule like hydrogen nearby to it, and the situation becomes favorable to adsorb molecular hydrogen when it approaches towards functionalized graphene. During this process of functionalization, there is not only the transfer of electronic charge but also the substrate itself develops dipole, quadrupole, and higher order of moment which helps to enhance the interaction of the material with a neutral molecule.

\section{Adsorption of hydrogen molecule/s on Co added grapheme}

One of the major tasks of the present work includes the adsorption of molecular hydrogen/s $\left(\mathrm{H}_{2}\right)$ on the suitable substrate, with suitable strength and density, basically in the range of DOE target (Zhou \& Williams, 2011). The pure graphene weakly binds molecular hydrogen through van der Wall's interaction, which is not favorable to adsorb and desorb hydrogen for energy storage at the operating conditions. The previous study of this group has shown enough evidence to use metal adatom in graphene (Na-graphene) to enhance adsorption energy towards gaseous hydrogen (Pantha et al., 2015a). In the present work, we have selected a single Co atom from the transition group of elements to functionalize $4 \times 4$ supercell of graphene, which gets polarized on graphene (as discussed above).

Fig. 6 presents the optimized structures for the adsorption of hydrogen molecules on the cobalt decorated graphene system. The minimum energy geometries display that two of the hydrogen molecules were dissociated for $\mathrm{n}=2$ and 3 , and they were strongly adsorbed to Co adatom graphene in the atomic form. Here the bond dissociation energy of hydrogen molecules was added to the adsorption energy of hydrogen on Co-adatom graphene (Xu et al., 2015). The strong binding of molecular hydrogen/s at low concentration is also supported by Table 2 and Fig. 7.
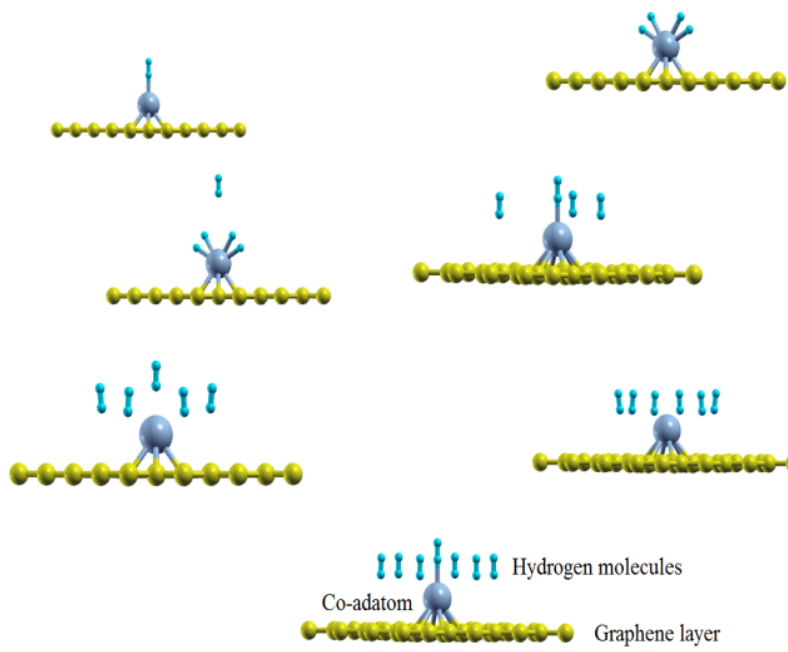

Fig. 6. Optimized geometry for the adsorption of the $\mathbf{H}_{2}$ molecules in the cobalt adsorbed graphene systems (for some hydrogen molecules, $n=1$ to 7 ). The plane of yellow atoms, larger blue atom, and small sky blue molecule/s represent the graphene layer, Co adatom, and hydrogen molecules, respectively

Table 2. Ground state energy of the $\mathrm{Co}-\mathrm{H}_{2}$-graphene, binding energy of $\mathrm{H}_{2}$ on the Co-added-graphene, and binding energy per $\mathrm{H}_{2}$ molecules, respectively

\begin{tabular}{cccc}
\hline $\begin{array}{c}\text { No. of } \mathrm{H}_{2} \\
\text { molecules }\end{array}$ & $\begin{array}{c}\text { Total energy } \\
(\mathrm{Ry})\end{array}$ & $\begin{array}{c}\text { Binding } \\
\text { energy }(\mathrm{eV})\end{array}$ & $\begin{array}{c}\text { Binding energy/ } \mathrm{H}_{2} \\
\text { molecule }(\mathrm{eV})\end{array}$ \\
\hline 1 & -441.266 & 0.353 & 0.353 \\
2 & -443.678 & 1.463 & 0.731 \\
3 & -446.011 & 1.668 & 0.556 \\
4 & -448.277 & 0.60 & 0.15 \\
5 & -450.615 & 0.703 & 0.141 \\
6 & -452.946 & 0.755 & 0.126 \\
7 & -455.288 & 0.798 & 0.114 \\
\hline
\end{tabular}


The total energy, adsorption energy for $\mathrm{n}$ hydrogen molecules, and binding energy per $\mathrm{H}_{2}$ molecule in cobalt decorated graphene are presented in Table 2. The adsorption energy for molecular hydrogens and adsorption energy per $\mathrm{H}_{2}$ are also shown in Fig. 7. They show that adsorption energy per hydrogen increases on increasing the number from 1 to 2 (high enough for $n=3$ also) and then decreases on further increasing the adsorbed number of $\mathrm{H}_{2}$ (except for $\mathrm{n}=7$ ). As discussed above (also shown by the geometry of Fig. 6), separation between the hydrogen atoms in a molecule significantly increases as if it dissociates into the atoms. Hence the bond dissociation energy between hydrogens adds up into its adsorption energy on the substrate. The London dispersion contribution, which is the dominant part of van der Waal's interaction in the case of gaseous adsorption (Ulman et al., 2014), has also been reported in Fig. 7. The figure shows almost constant values of dispersion contribution (in the order of $0.08 \mathrm{eV}$ ) as a function of the number of hydrogen molecules. For the higher number of $\mathrm{H}_{2}$, the adsorption energy seems to be contributed mainly by the dispersion corrections. The small difference between them (adsorption energy and dispersion correction) can be understood due to other types of van der Waal's interactions, like Debye and Keesom contributions (Klimes \& Michaelides, 2012), which are not included in the present work. However, it has already been reported that DFT-D2, which only incorporates the London dispersion correction, predicts good accuracy of van der Waal's interactions (Joshi \& Ghosh, 2013).

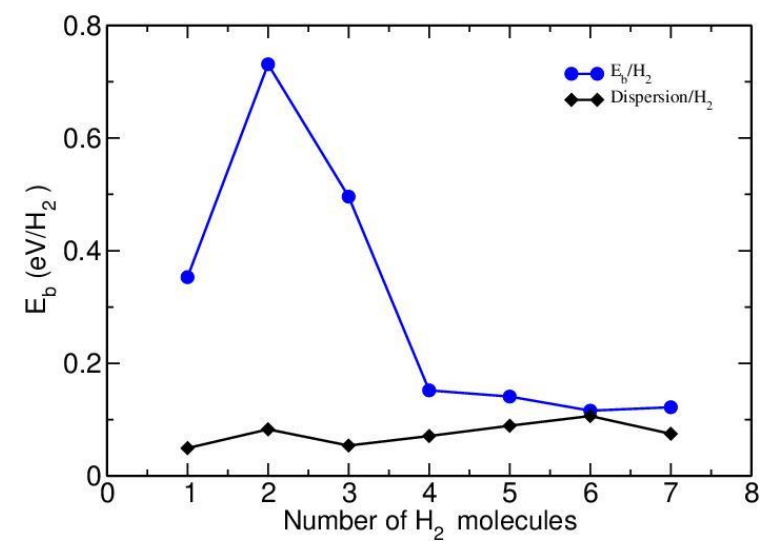

Fig. 7. Variation of binding energy (used binding energy and adsorption energy as synonyms) and dispersion contribution to binding energy per $\mathrm{H}_{2}$ molecule as a function of the number of hydrogen molecules adsorbed in cobalt adsorbed graphene

The charge transfer phenomenon (for $n=1,2$, and 3 ) due to molecular adsorption of hydrogen/s on functionalized graphene (Figs. $8 \&$ 9) has also been considered. During the process of calculations, the method of planner average of electronic charge density difference has been followed. The electronic charge density difference between the product compound and its constituents quantifies the rearrangement of the charge (accumulation or depletion) in the given plane. Then one can define the cut-off point $\mathrm{R}_{\text {cut }}$ from which charge accumulation changes to charge depletion (or vice-versa). The integration above (below) $\mathrm{R}_{\text {cut }}$ along the $\mathrm{z}$-axis gives the total electronic charge that transfers from (towards) the molecular hydrogens (Chan et al., 2008). Present calculations found the electronic charge transfer from hydrogen/s towards the substrate as $0.005 \mathrm{e}, 1.077 \mathrm{e}$, and $0.377 \mathrm{e}$ respectively for the number of hydrogen molecules $(\mathrm{n}=1,2$, and 3 ). The magnitude of charge transfer (1.077e) during the adsorption of two hydrogen molecules means that almost one electron transfers from the hydrogen. It indicates the possibility of ionic-dominant bonding between them. Also, the adsorption of three hydrogen molecules shows a significant charge transfer $(0.377 \mathrm{e})$. This number and the geometry explain the higher adsorption energy/ $\mathrm{H}_{2}$ for a low number of molecular hydrogen.

The charge lobes (green and red) of electronic charge (Fig. 8) show charge depletion and accumulation during the interaction. The green (red) lobes represent the charge depleted (accumulated) regions. The green lobes towards the region of molecular hydrogen/s (at the far end from the adatom) indicate that electronic charge transfers from the gaseous molecules towards the substrate. Since the charge was transferred from adatom (Co) towards the graphene layer (discussed above) during its adsorption, deficiency of electron/s in cobalt makes it more positive and attracts electronic charge from the hydrogen.

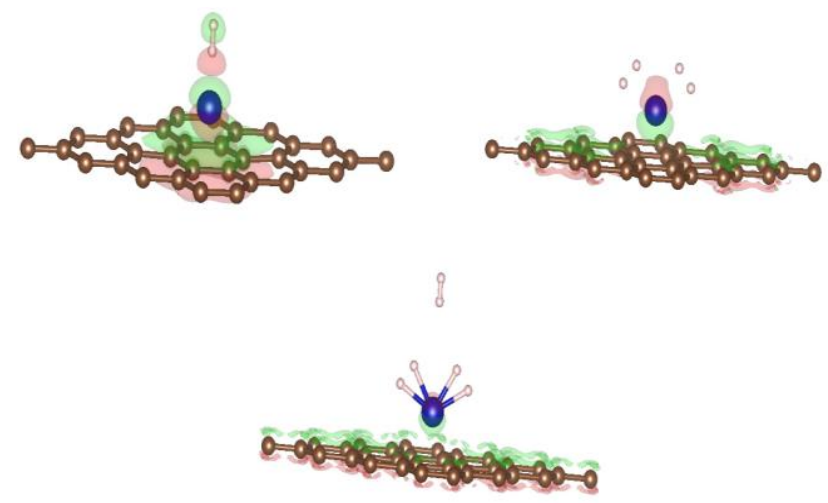

Fig. 8. Charge density difference calculations for hydrogen molecule $(n=1,2,3)$ adsorbed on cobalt atom added graphene of size $4 \times 4$. The green lobes (red lobes) represent the charge depletion (accumulation) regions in the isosurface structures (color online)

The stronger binding strength towards the lower number of hydrogen molecules can also be described with the coordination number (number of nearest neighbors to the reference atom). When the number of hydrogen/s is low, they form strong (chemical) bonding with cobalt atom either by sharing of electron/s or by transfer of electrons 
or by both the ways, as the constituents (cobalt and hydrogen) have partially filled electronic orbitals. The process of this type of interaction between the transition metal atom and molecular hydrogen has been described in terms of Kubas interaction (Skipper et al., 2012). Once the electronic orbitals are filled, there are no vacancies to accommodate more hydrogens for chemical bonding. Hence the additional hydrogen molecules beyond some number are weakly bound either by physisorption or in excited mode (Pantha et al., 2015b). Here two molecules of hydrogen seem to complete the demand of electronic charge for the formation of cobalt metal-hydride.

The adsorption of hydrogen as a process of energy storage is superior over the traditional methods like liquefied natural gases (LNG) and compressed natural gases (CNG) due to safety, space, economy, and technological perspective. The department of energy, United States (DOE, US) has set two major targets for its practical applications, i) in the sense of energy density (about $6 \mathrm{wt}$ $\%$ of $\mathrm{H}_{2}$ ) and ii) storage and release at operating conditions (adsorption energy 0.2 to $0.7 \mathrm{eV}$ ) (Durgun et al., 2008). The present work found an appropriate order of adsorption energy per $\mathrm{H}_{2}$. Regarding the content of hydrogen, this work found $3.061 \%$ by weight, and there are several possible ways to improve the material so that the Co-functionalized graphene can hold more hydrogen molecules.

Fig. 9 shows the planar average method of calculations for finding charge transfer from one constituent (hydrogen) to another constituent (metal-graphene) of the complex. In the first figure, the positions for modeled cobalt and hydrogen are shown. The black and red lines (color online) represent the regime of graphene and adatomhydrogen/s whereas the vertical dotted line represents (Rcut) the plane which separates the region for hydrogen/s from the substrate. The integration above/below this dotted vertical line gives the magnitude of charge transfer.

\section{CONCLUSIONS}

The most stable geometry of functionalized graphene, with single Co atom, has been predicted by using DFT level of calculations in quantum espresso codes. The binding energy values at varying size graphene supercell show that the hollow site is the most favorable place to adsorb Co. The band structure calculations for Co-adatom graphene displayed the overlapping nature of conduction band and valence band, and also the shifting up of Fermi level from the conical point. The density of states (DOS) calculations revealed that spin up and spin down states were not symmetrical which indicated that the cobalt decorated graphene system is magnetic due to the contribution of $4 \mathrm{~s}$ and $3 \mathrm{~d}$ orbitals of the cobalt atom. Regarding the case of molecular adsorption of hydrogen/s on the cobalt decorated graphene, the adsorption energy per hydrogen molecule was enhanced compared to that in pristine graphene. The adsorption energy depends on the type of interaction, basically in between the gaseous hydrogen and the substrate. The present work quantified the adsorption energy in the order of $(0.116-0.731)$ $\mathrm{eV} / \mathrm{H}_{2}$. Most of these values met the DOE target for the adsorption of gaseous fuel.
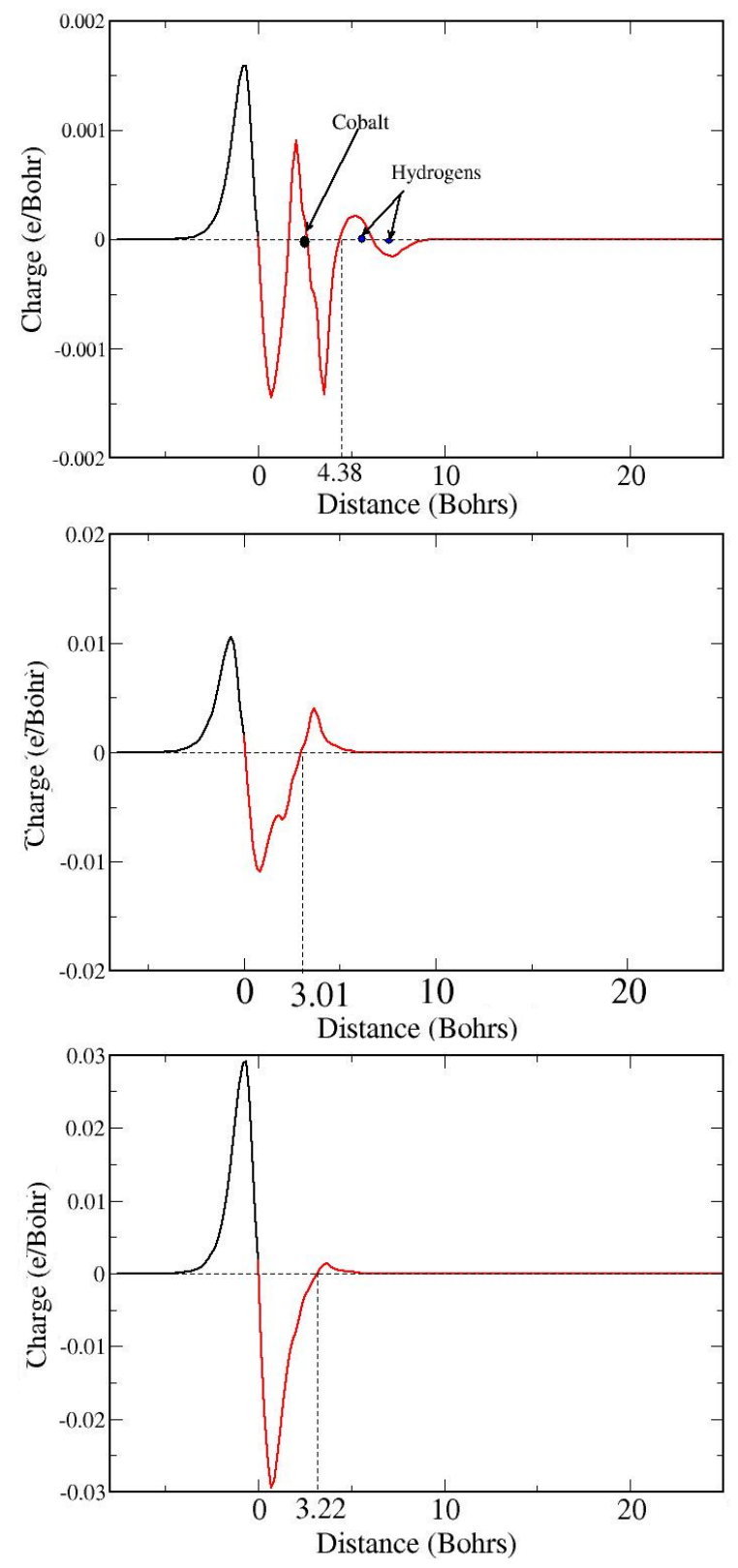

Fig. 9. Planar averaged electronic charge density difference for hydrogen/s ( $=1,2$, and 3) on cobalt decorated graphene at $\mathrm{H}$ site as a function of position in the z-direction

\section{ACKNOWLEDGMENTS}

NP and NPA acknowledge the University Grants Commission Nepal Award no. CRG-73/74-S\&T-01. 


\section{REFERENCES}

Bhatia, S. K., \& Myers, A. L. (2006). Optimum conditions for adsorptive storage. Langmuir, 22(4), 1688-1700.

Chan, K. T., Neaton, J. B., \& Cohen, M. L. (2008). Firstprinciples study of metal adatom adsorption on graphene. Physical Review B, 77(23), 235430 (12pp).

Chen, P., Wu, X., Lin, J., \& Tan, K. L. (1999). High $\mathrm{H}_{2}$ uptake by alkali-doped carbon nanotubes under ambient pressure and moderate temperature. Science, 285(5424), 91-93.

Dahal, S., Kafle, G., Kaphle, G. C., \& Adhikari, N. P. (2014). Study of electronic and magnetic properties of $\mathrm{CuPd}, \mathrm{CuPt}, \mathrm{Cu} 3 \mathrm{Pd}$, and Cu3Pt: tight-binding linear muffin-tin orbitals approach. Journal of Institute of Science and Technology, 19(1), 137-144.

Durgun, E., Ciraci, S., \& Yildirim, T. (2008). Functionalization of carbon-based nanostructures with light transition-metal atoms for hydrogen storage. Physical Review B, 77(8), 085405 (9pp).

Geim, A. K. (2009). Graphene: status and prospects. Science, 324(5934), 1530-1534.

Geim, A. K., \& Novoselov, K. S. (2007). The rise of graphene. Nature Materials, 6, 183-191.

Giannozzi, P., Baroni, S., Bonini, N., Calandra, M., Car, R., Cavazzoni, C., Ceresoli, D., Chiarotti, G. L., Cococcioni, M., Dabo, I., Carso, A. D., Girancoli, S., Fabris, S., Fratesi, G., Gebauer, R., Gerstmann, U., Gougoussis, C., Kokalj, A., Lazzeri, M., MartinSamos, L., Marzari, N., Mauri, F., Mazzarello, R., Paolini, S., Pasquarello, A., Paulatto, L., Sbraccia, C., Scandolo, S., Sclauzero, G., Seitsonen, A. P., Smogunov, A., Umari, P., \& Wintzcovitch, R. M., (2009). QUANTUM ESPRESSO: a modular and open-source software project for quantum simulations of materials. Journal of Physics: Condensed Matter, 21(39), 395502 (19pp).

Han, M. Y., Özyilmaz, B., Zhang, Y., \& Kim, P. (2007). Energy band-gap engineering of graphene nanoribbons. Physical Review Letters, 98(20), 206805 (4pp).

Head J. D., \& Zerner M. C. (1985). A Broyden-FletcherGoldfarb-Shanno optimization procedure for molecular geometries, Chemical Physics Letters, 122(3), 264-270.

Hohenberg, P., \& Kohn, W. (1964). Density functional theory (DFT). Physical Review, 136, B864-B871.
Jain, V., \& Kandasubramanian, B., (2020). Functionalized graphene materials for hydrogen storage. Journal of Materials Science, 55, 1865-1903.

Joshi, N., \& Ghosh, P. (2013). Substrate-induced changes in the magnetic and electronic properties of hexagonal boron nitride. Physical Review B, 87(23), 235440 (8pp).

Kittel, C. (2015). Introduction to solid state physics $\left(8^{\text {th }}\right.$ ed.), Daryaganj, India: Wiley India Pvt. Ltd.

Klimeš, J., \& Michaelides, A. (2012). Perspective: Advances and challenges in treating van der Waals dispersion forces in density functional theory. The Journal of Chemical Physics, 137, 120901 (12pp).

Kohn, W., \& Sham, L. J., (1965). Self-consistent equations including exchange and correlation effect. Physical Review 140, A1133-A1134.

Marzari, N., Vanderbilt, D., De Vita, A., \& Payne, M. C. (1999). Thermal contraction and disordering of the Al(110) surface. Physical Review Letters, 82(16), 3296-3299.

Monkhorst, H. J., \& Pack, J. D. (1976). Special points for Brillouin-zone integrations. Physical Review B, 13(12), 5188-5192.

Neto, A. C., Guinea, F., \& Peres, N. M. (2006). Drawing conclusions from graphene. Physics World, 19(11), 33-37.

Neto, A. C., Guinea, F., Peres, N. M., Novoselov, K. S., \& Geim, A. K. (2009). The electronic properties of graphene. Reviews of Modern Physics, 81(1), 109162.

Novoselov, K. S., Geim, A. K., Morozov, S., Jiang, D., Katsnelson, M. I., Grigorieva, I., ... \& Firsov, A. A. (2005). Two-dimensional gas of massless Dirac fermions in graphene. Nature, 438(7065), 197-200.

Pan, L., Sander, M. B., Huang, X., Li, J., Smith, M., Bittner, E., ... \& Johnson, J. K. (2004). Microporous metal organic materials: promising candidates as sorbents for hydrogen storage. Journal of the American Chemical Society, 126(5), 1308-1309.

Pantha, N., Belbase, K., and Adhikari, N. P. (2015a). First-principles study of interaction of hydrogen molecular on Na-adsorbed graphene, Applied Nanoscience, 5, 393-502.

Pantha, N., \& Adhikari, N. P. (2014). Structure and symmetrization of hydrogen bonding in ices VIII and $\mathrm{X}$ at high pressure: a density functional theory approach. Journal of Institute of Science and Technology, 19(2), 14-19. 
Pantha, N., Khaniya, A., \& Adhikari, N.P. (2015b). Hydrogen storage on palladium adsorbed graphene: a density functional theory study. International Journal of Modern Physics B, 29(20), 1550143 (14pp).

Paudel, B. P., Pantha, N., \& Adhikari, N. P. (2015). Firstprinciples study of electronic and magnetic properties of manganese decorated graphene. Journal of Nepal Physical Society, 2(1), 33-43.

Paudel, S., Adhikari, P. R., Upadhyay, O. P., Kaphle, G. C., \& Srivastava, A., (2018). First-principles study of electronic and optical properties of pristine and $\mathrm{X}(\mathrm{Cu}, \mathrm{Ag}$, and $\mathrm{Au})$ doped $\mathrm{BiOBr}$. Journal of Institute of Science and Technology, 22(2), 63-69.

Perdew, J. P., Burke, K., \& Ernzerhof, M. (1996). Generalized gradient approximation made simple. Physical Review Letters, 77(18), 3865-3868.

Sevinçli, H., Topsakal, M., Durgan, E., \& Ciraci, S. (2008). Electronic and magnetic properties of 3dtransition metal atom adsorbed graphene and graphene nanoribbons. Physical Review B, 77, $195434(7 \mathrm{pp})$.

Skipper, C.V..J., Hamaed, A., Antonelli, D.M., \& Kaltsoyannis, N., (2012). The Kubas interaction in $\mathrm{M}(\mathrm{II})(\mathrm{M}=\mathrm{Ti}, \mathrm{V}, \mathrm{Cr})$ hydrazine-based hydrogen storage materials: a DFT study. Dalton Transactions, $41,8515-8523$.

Ulman, K., Bhaumik, D., Wood, B. C., \& Narasimhan, S. (2014). Physical origins of weak $\mathrm{H}_{2}$ binding on carbon nanostructures: insight from ab initio studies of chemically functionalized graphene nanoribbons. The Journal of chemical physics, 140(17), 174708 (12pp).

Valencia, H., Gil, A., \& Frapper, G. (2010). Trends in the adsorption of $3 \mathrm{~d}$ transition metal atoms onto graphene and nanotube surfaces: a DFT study and molecular orbital analysis. The Journal of Physical Chemistry C, 114(33), 14141-14153.

Xu, C., Brown, P.A., Lu J. \& Shuford K. L. (2015). Electronic properties of halogen-adsorbed graphene. The Journal of Physical Chemistry C, 119, 1727117277.

Yagi, Y., Briere, T. M., Sluiter, M. H., Kumar, V., Farajian, A. A., \& Kawazoe, Y. (2004). Stable geometries and magnetic properties of single-walled carbon nanotubes doped with $3 \mathrm{~d}$ transition metals: A first-principle study. Physical Review B, 69(7), 075414 (9pp).

Zhang, L., Cai, Z., Yao, Z., Ji, L., Sun, Z., Yan, N., Zhang, B., Xiao, B., Du, J., Zhu, X., \& Chen, L. (2019). A striking catalytic effect of facile synthesized $\mathrm{ZrMn}_{2}$ nanoparticles on the de/rehydrogenation properties of $\mathrm{MgH}_{2}$. Journal of Materials Chemistry A, 7(10), 5626-5634.

Zhou, J. G., \& Williams, Q. L. (2011). Hydrogen storage on platinum-decorated carbon nanotubes with boron, nitrogen dopants, or sidewall vacancies. Journal of Nano Research, 15, 29-40. 